QUARTERLY OF APPLIED MATHEMATICS

VOLUME LXV, NUMBER 1

MARCH 2007, PAGES 135-143

S $0033-569 \mathrm{X}(06) 01043-6$

Article electronically published on December 14, 2006

\title{
LARGE TIME DECAY OF SOLUTIONS TO ISENTROPIC GAS DYNAMICS
}

\author{
BY
}

\author{
NAOKI TSUGE
}

\begin{abstract}
Department of Pure and Applied Mathematics, Graduate School of Information Science and Technology, Osaka University, Osaka 560-0043, Japan
\end{abstract}

Abstract. We study the large time behavior of solutions to isentropic gas dynamics. For a constant $\gamma(1<\gamma<3)$, we show the decay of the $L^{\gamma}$ norm of density. To do this, we estimate approximate solutions constructed by a difference scheme.

1. Introduction. We study isentropic gas dynamics:

$$
\left\{\begin{array}{l}
\rho_{t}+m_{x}=0 \\
m_{t}+\left(\frac{m^{2}}{\rho}+p(\rho)\right)_{x}=0
\end{array}\right.
$$

where $\rho, m$ and $p$ are density, momentum and pressure of gas, respectively. For a nonvacuum state $\rho>0, u=m / \rho$ is velocity. For polytropic gas, $p(\rho)=\rho^{\gamma} / \gamma$, where $\gamma$ is a constant satisfying $1<\gamma<3$.

Then we consider the Cauchy problem (1.1) with initial data:

$$
\left.(\rho, m)\right|_{t=0}=\left(\rho_{0}(x), m_{0}(x)\right) .
$$

By using a vector $v={ }^{t}(\rho, m)$, (1.1) -1.2 can be written as

$$
\left\{\begin{array}{l}
v_{t}+f(v)_{x}=0 \\
\left.v\right|_{t=0}=v_{0}(x)
\end{array}\right.
$$

where $f(v)={ }^{t}\left(m, m^{2} / \rho+p(\rho)\right)$.

Now, to state the main theorem in this paper, let us define the Riemann invariants $w, z$, mechanical energy $\eta(v)$ and its flux $q(v)$ as follows:

$$
\begin{aligned}
& w:=\frac{m}{\rho}+\frac{\rho^{\theta}}{\theta}=u+\frac{\rho^{\theta}}{\theta}, z:=\frac{m}{\rho}-\frac{\rho^{\theta}}{\theta}=u-\frac{\rho^{\theta}}{\theta} \quad\left(\theta:=\frac{\gamma-1}{2}\right), \\
& \eta(v):=\frac{m^{2}}{\rho}+\frac{p(\rho)}{\theta}, \quad q(v):=\left(\frac{m^{2}}{\rho}+\frac{\gamma p(\rho)}{\theta}\right) \frac{m}{\rho} .
\end{aligned}
$$

Received March 30, 2006.

2000 Mathematics Subject Classification. Primary 35L65, 35L60, 76J20.

Key words and phrases. Isentropic gas dynamics, decay, the Lax-Friedrichs scheme.

The author was supported in part by JSPS Research Fellowships for Young Scientists.

E-mail address: tuge@math.sci.osaka-u.ac.jp 
Then our main theorem is as follows:

Theorem 1.1. We assume that, for $B \geq 0$ and $C \geq 0$, initial data (1.2) satisfy

$$
\begin{aligned}
& -B \leq z\left(v_{0}(x)\right), \quad w\left(v_{0}(x)\right) \leq B, \\
& \int_{-\infty}^{\infty} x^{2} \rho_{0}(x) d x \leq C, \quad \int_{-\infty}^{\infty} \eta\left(v_{0}(x)\right) d x \leq C .
\end{aligned}
$$

Then, for a.e. $t>1$, a solution to (1.1) -(1.2) as the limit of the Lax-Friedrichs approximate solutions satisfies

$$
\int_{-\infty}^{\infty}\{\rho(x, t)\}^{\gamma} d x \leq \frac{\gamma \theta}{t^{\gamma-1}}\left(\int_{-\infty}^{\infty} x^{2} \rho_{0}(x) d x+\frac{3-\gamma}{2} \int_{-\infty}^{\infty} \eta\left(v_{0}(x)\right) d x\right) .
$$

In [4, this type of decay is discussed by using a kinetic formulation. On the other hand, the global existence of a solution to (1.3) is proved by the Lax-Friedrichs scheme and compensated compactness in [2]. In this paper, we show the decay of the solutions obtained by the Lax-Friedrichs scheme. In Section 2, we state the Rankine-Hugoniot condition, the entropy condition and the theory of invariant regions for the Riemann problem. In Section 3, we construct approximate solutions by the Lax-Friedrichs scheme and investigate their bounds, mass and energy. In Section 4, we derive decay estimates of the approximate solutions to prove Theorem 1.1 .

2. Preliminary. Discontinuous solutions arise for (1.1). The jump discontinuity in a weak solution to (1.1) must satisfy the following Rankine-Hugoniot condition:

$$
\lambda\left(v-v_{0}\right)=f(v)-f\left(v_{0}\right),
$$

where $\lambda$ is the propagation speed of the discontinuity, and $v_{0}=\left(\rho_{0}, m_{0}\right)$ and $v=(\rho, m)$ are the corresponding left and right states respectively. Furthermore a jump discontinuity is called a shock if it satisfies the entropy condition:

$$
\lambda\left(\eta(v)-\eta\left(v_{0}\right)\right)-\left(q(v)-q\left(v_{0}\right)\right) \geq 0 .
$$

Next, let us recollect the Riemann problem for (1.1) with initial data

$$
\left.v\right|_{t=0}= \begin{cases}v_{-}, & x<x_{0} \\ v_{+}, & x>x_{0}\end{cases}
$$

where $\rho_{ \pm} \geq 0$ and $m_{ \pm}$are constants satisfying $\left|m_{ \pm}\right| \leq C \rho_{ \pm}$. The solution to (1.1) with (2.3) consists of rarefaction waves and shocks. We call this solution the Riemann solution. The Riemann solution has the following properties:

Lemma 2.1. For $\mathrm{B}_{+} \geq \mathrm{B}_{-}$, the region $\sum\left(\mathrm{B}_{+}, \mathrm{B}_{-}\right)=\left\{(\rho, \rho u) \in \mathbf{R}^{2}: w=u+\rho^{\theta} / \theta, z=\right.$ $\left.u-\rho^{\theta} / \theta, w \leq \mathrm{B}_{+}, z \geq \mathrm{B}_{-}, w-z \geq 0\right\}$ is invariant with respect to both of the Riemann problem (1.1) with (2.3) and the average of the Riemann solutions in $x$. More precisely, if the Riemann data lie in $\sum\left(\mathrm{B}_{+}, \mathrm{B}_{-}\right)$, the corresponding Riemann solutions $(\rho(x, t), m(x, t))=(\rho(x, t), \rho(x, t) u(x, t))$ lie in $\sum\left(\mathrm{B}_{+}, \mathrm{B}_{-}\right)$, and their corresponding averages in $x$ also lie in $\sum\left(\mathrm{B}_{+}, \mathrm{B}_{-}\right)$, namely

$$
\left(\frac{1}{b-a} \int_{a}^{b} \rho(x, t) d x, \frac{1}{b-a} \int_{a}^{b} m(x, t) d x\right) \in \sum\left(\mathrm{B}_{+}, \mathrm{B}_{-}\right) .
$$


The proof of Lemma 2.1 can be found in [2, Lemma 3.3].

3. Lax-Friedrichs approximate solutions. In this section, we first introduce the Lax-Friedrichs scheme and construct the Lax-Friedrichs approximate solutions. We next investigate their bounds, mass and energy.

3.1. The Lax-Friedrichs scheme. In this subsection, we construct approximate solutions by the Lax-Friedrichs scheme. Let us denote the approximate solutions by $v^{\Delta}(x, t)=\left(\rho^{\Delta}(x, t), m^{\Delta}(x, t)\right)$. In addition, we choose the space mesh length $\Delta x$ and the time mesh length $\Delta t$ satisfying $0<\Delta t<1$ and the following Courant-Friedrichs-Lewy condition:

$$
\Lambda:=\max _{i=1,2}\left(\sup _{0 \leq t, x \in \mathbf{R}}\left|\lambda_{i}\left(v^{\Delta}(x, t)\right)\right|\right) \leq \frac{\Delta x}{\Delta t} \leq 2 \Lambda,
$$

where $\lambda_{1}:=u-\rho^{\theta}, \lambda_{2}:=u+\rho^{\theta}$.

Now we construct the approximate solution $v^{\Delta}(x, t)$. Let

$$
(j, n) \in \mathbf{Z} \times \mathbf{Z}_{\geq 0}, \quad J_{n}=\{j \in \mathbf{Z}: n+j=\text { even }\} .
$$

First, we define

$$
v^{\Delta}(x,-0)=v_{0}(x) \chi^{\Delta}(x)
$$

where

$$
\chi^{\Delta}(x)= \begin{cases}1, & x \in[-\alpha(1 / \Delta x), \alpha(1 / \Delta x)] \\ 0, & \text { otherwise }\end{cases}
$$

$\alpha: \mathbf{R}_{+} \mapsto \mathbf{R}_{+}$is a smooth function such that $\alpha(x) \rightarrow \infty$ as $x \rightarrow \infty$.

Second, we assume that $v^{\Delta}(x, t)$ is defined for $t<n \Delta t$. Then we define $v_{j}^{n}:=\left(\rho_{j}^{n}, m_{j}^{n}\right)$ as, for $j \in J_{n}$,

$$
\left\{\begin{array}{l}
\rho_{j}^{n}:=\frac{1}{2 \Delta x} \int_{(j-1) \Delta x}^{(j+1) \Delta x} \rho^{\Delta}(x, n \Delta t-0) d x, \quad(j-1) \Delta x \leq x \leq(j+1) \Delta x, \\
m_{j}^{n}:=\frac{1}{2 \Delta x} \int_{(j-1) \Delta x}^{(j+1) \Delta x} m^{\Delta}(x, n \Delta t-0) d x, \quad(j-1) \Delta x \leq x \leq(j+1) \Delta x .
\end{array}\right.
$$

Finally, in the strip $n \Delta t \leq t<(n+1) \Delta t, v^{\Delta}(x, t)$ is defined as, for $j \Delta x \leq x<(j+2) \Delta x$, the solution of the Riemann problem at $x=(j+1) \Delta x$ :

$$
\left\{\begin{array}{l}
v_{t}+f(v)_{x}=0, \quad j \Delta x \leq x<(j+2) \Delta x, \\
\left.v\right|_{t=n \Delta t}= \begin{cases}v_{j}^{n}, & x<(j+1) \Delta x, \\
v_{j+2}^{n}, & x>(j+1) \Delta x .\end{cases}
\end{array}\right.
$$

This completes the definition of approximate solutions.

3.2. Bounds, mass and energy of Lax-Friedrichs approximate solutions. In this subsection, we estimate the approximate solutions defined above. Our goal is to derive some estimates, which shall be used in the next section. First we observe that the approximate solutions satisfy the following:

(i) From (1.4) and Lemma 2.1

$$
-B \leq z\left(v^{\Delta}(x, t)\right), \quad w\left(v^{\Delta}(x, t)\right) \leq B .
$$


(ii) For $j \in J_{n+1}$,

$$
\begin{aligned}
& \rho_{j}^{n+1}=\frac{\rho_{j+1}^{n}+\rho_{j-1}^{n}}{2}-\frac{\Delta t}{2 \Delta x}\left(m_{j+1}^{n}-m_{j-1}^{n}\right), \\
& m_{j}^{n+1}=\frac{m_{j+1}^{n}+m_{j-1}^{n}}{2}-\frac{\Delta t}{2 \Delta x}\left\{\frac{\left(m_{j+1}^{n}\right)^{2}}{\rho_{j+1}^{n}}+p\left(\rho_{j+1}^{n}\right)-\frac{\left(m_{j-1}^{n}\right)^{2}}{\rho_{j-1}^{n}}-p\left(\rho_{j-1}^{n}\right)\right\}
\end{aligned}
$$

and

$$
\eta\left(v_{j}^{n+1}\right) \leq \frac{\eta\left(v_{j+1}^{n}\right)+\eta\left(v_{j-1}^{n}\right)}{2}-\frac{\Delta t}{2 \Delta x}\left(q\left(v_{j+1}^{n}\right)-q\left(v_{j-1}^{n}\right)\right) .
$$

From (i)-(ii) and the assumption of initial data, we have the following lemma:

LEmma 3.1. The Lax-Friedrichs approximate solutions defined above satisfy the following:

(1) Bounds: From (3.2), we have

$$
\left\{\rho^{\Delta}(x, t)\right\}^{\theta} / \theta \leq B, \quad-B \leq u^{\Delta}(x, t) \leq B, \quad \Lambda \leq B .
$$

(2) Mass: From (3.3), we have

$$
\sum_{j \in J_{n+1}} \int_{(j-1) \Delta x}^{(j+1) \Delta x} \rho_{j}^{n+1} d x=\sum_{j \in J_{n}} \int_{(j-1) \Delta x}^{(j+1) \Delta x} \rho_{j}^{n} d x .
$$

Furthermore, there exists $M_{B, C}$ depending only on $B$ and $C$ such that

$$
\begin{gathered}
\sum_{j \in J_{n}} \int_{(j-1) \Delta x}^{(j+1) \Delta x} \rho_{j}^{n} d x \leq M_{B, C} . \\
\sum_{j \in J_{0}} \int_{(j-1) \Delta x}^{(j+1) \Delta x} x^{2} \rho_{j}^{0} d x \leq \int_{\infty}^{\infty} x^{2} \rho_{0}(x) d x+O(\Delta x),
\end{gathered}
$$

where $O(\Delta x)$ depends only on $B$ and $C$.

(3) Energy: From (3.5), we have

$$
\sum_{j \in J_{n+1}} \int_{(j-1) \Delta x}^{(j+1) \Delta x} \eta\left(v_{j}^{n+1}\right) d x \leq \sum_{j \in J_{n}} \int_{(j-1) \Delta x}^{(j+1) \Delta x} \eta\left(v_{j}^{n}\right) d x
$$

and (by using the Jensen inequality)

$$
\sum_{j \in J_{0}} \int_{(j-1) \Delta x}^{(j+1) \Delta x} \eta\left(v_{j}^{0}\right) d x \leq \int_{-\infty}^{\infty} \eta\left(v_{0}(x)\right) d x \leq C .
$$

4. Decay estimates of the Lax-Friedrichs approximate solutions. In this section, we derive decay estimates of the Lax-Friedrichs approximate solutions defined in the previous section. Through this paper, by Landau's symbols such as $O(\Delta x)$ and $O\left((\Delta x)^{2}\right)$, we denote quantities whose moduli satisfy uniform bounds depending only on $B$ and $C$. 
4.1. Estimates of $v_{j}^{n}$. First we define

$$
\begin{aligned}
& F(x, t, v)=x^{2} \rho-2 x t m+t^{2} \eta(v), \\
& G(x, t, v)=x^{2} m-2 x t\left(m^{2} / \rho+p(\rho)\right)+t^{2} q(v), \\
& H(x, t, v)=2 t(1 / \theta-1) p(\rho) .
\end{aligned}
$$

Then, our goal in this subsection is to prove the following lemma:

Lemma 4.1. We choose any integer $N$ such that $N \Delta t \geq 1$. Furthermore, let $N_{1}$ be the smallest integer such that $N_{1} \Delta t \geq 1$. Then we have

$$
\begin{aligned}
& \sum_{j \in J_{N}} \int_{(j-1) \Delta x}^{(j+1) \Delta x}\{N \Delta t\}^{\gamma-3} F\left(x, N \Delta t, v_{j}^{N}\right) d x \\
& \quad \leq \int_{-\infty}^{\infty} x^{2} \rho_{0}(x) d x+(3-\gamma) \frac{N_{1}\left(N_{1}-1\right)}{2}(\Delta t)^{2} \int_{-\infty}^{\infty} \eta\left(v_{0}(x)\right) d x+N \Delta t O(\Delta x) .
\end{aligned}
$$

Proof. Step 1. First we assume the following inequality holds:

$$
\begin{aligned}
& \sum_{j \in J_{n+1}} \int_{(j-1) \Delta x}^{(j+1) \Delta x} F\left(x,(n+1) \Delta t, v_{j}^{n+1}\right) d x \\
& \leq \sum_{j \in J_{n}} \int_{(j-1) \Delta x}^{(j+1) \Delta x} F\left(x, n \Delta t, v_{j}^{n}\right) d x+\sum_{j \in J_{n}} \int_{(j-1) \Delta x}^{(j+1) \Delta x} \Delta t H\left(x, n \Delta t, v_{j}^{n}\right) d x+O\left((\Delta x)^{2}\right) .
\end{aligned}
$$

The proof of (4.1) is given in the next step.

Then, from the inequality above and (3.10), we deduce that

$$
\begin{aligned}
\sum_{j \in J_{N_{1}}} & \int_{(j-1) \Delta x}^{(j+1) \Delta x} F\left(x, N_{1} \Delta t, v_{j}^{N_{1}}\right) d x \\
\leq & \sum_{j \in J_{0}} \int_{(j-1) \Delta x}^{(j+1) \Delta x} F\left(x, 0, v_{j}^{0}\right) d x+\sum_{n=0}^{N_{1}-1} \sum_{j \in J_{n}} \int_{(j-1) \Delta x}^{(j+1) \Delta x} \Delta t H\left(x, n \Delta t, v_{j}^{n}\right) d x \\
& +N_{1} \Delta t O(\Delta x) \\
\leq & \sum_{j \in J_{0}} \int_{(j-1) \Delta x}^{(j+1) \Delta x} F\left(x, 0, v_{j}^{0}\right) d x+(3-\gamma) \sum_{n=0}^{N_{1}-1} \sum_{j \in J_{n}} \int_{(j-1) \Delta x}^{(j+1) \Delta x} n(\Delta t)^{2} \eta\left(x, n \Delta t, v_{j}^{n}\right) d x \\
& +N_{1} \Delta t O(\Delta x) \\
\leq & \sum_{j \in J_{0}} \int_{(j-1) \Delta x}^{(j+1) \Delta x} F\left(x, 0, v_{j}^{0}\right) d x+(3-\gamma) \sum_{n=0}^{N_{1}-1} n(\Delta t)^{2} \sum_{j \in J_{0}} \int_{(j-1) \Delta x}^{(j+1) \Delta x} \eta\left(x, 0, v_{j}^{0}\right) d x \\
& +N_{1} \Delta t O(\Delta x) \\
\leq & \sum_{j \in J_{0}} \int_{(j-1) \Delta x}^{(j+1) \Delta x} F\left(x, 0, v_{j}^{0}\right) d x+(3-\gamma) \frac{N_{1}\left(N_{1}-1\right)}{2}(\Delta t)^{2} \sum_{j \in J_{0}} \int_{(j-1) \Delta x}^{(j+1) \Delta x} \eta\left(x, 0, v_{j}^{0}\right) d x \\
& +N_{1} \Delta t O(\Delta x) .
\end{aligned}
$$


On the other hand, for an integer $n$ such that $n \Delta t \geq 1$, multiplying both sides of (4.1) by $((n+1) \Delta t)^{\gamma-3}$, we have

$$
\begin{aligned}
& \sum_{j \in J_{n+1}} \int_{(j-1) \Delta x}^{(j+1) \Delta x}((n+1) \Delta t)^{\gamma-3} F\left(x,(n+1) \Delta t, v_{j}^{n+1}\right) d x \\
& \leq \sum_{j \in J_{n}} \int_{(j-1) \Delta x}^{(j+1) \Delta x}((n+1) \Delta t)^{\gamma-3}\left(x-n \Delta t u_{j}^{n}\right)^{2} \rho_{j}^{n} d x \\
& \quad+\sum_{j \in J_{n}} \int_{(j-1) \Delta x}^{(j+1) \Delta x}((n+1) \Delta t)^{\gamma-3}(n \Delta t)^{2} p\left(\rho_{j}^{n}\right) / \theta d x \\
& \quad+\sum_{j \in J_{n}} \int_{(j-1) \Delta x}^{(j+1) \Delta x}((n+1) \Delta t)^{\gamma-3} \Delta t H\left(x, n \Delta t, v_{j}^{n}\right) d x+O\left((\Delta x)^{2}\right) \\
& \leq \sum_{j \in J_{n}} \int_{(j-1) \Delta x}^{(j+1) \Delta x}(n \Delta t)^{\gamma-3}\left(x-n \Delta t u_{j}^{n}\right)^{2} \rho_{j}^{n} d x \\
& \quad+\sum_{j \in J_{n}} \int_{(j-1) \Delta x}^{(j+1) \Delta x}(n \Delta t)^{\gamma-3}(n \Delta t)^{2} p\left(\rho_{j}^{n}\right) / \theta d x+O\left((\Delta x)^{2}\right) \\
& =\sum_{j \in J_{n}} \int_{(j-1) \Delta x}^{(j+1) \Delta x}(n \Delta t)^{\gamma-3} F\left(x, n \Delta t, v_{j}^{n}\right) d x+O\left((\Delta x)^{2}\right) .
\end{aligned}
$$

Here, for the second term on the right-hand side of the first inequality (resp. the first and third terms on the right-hand side of the first inequality), we have used $((n+1) \Delta t)^{\gamma-3}=$ $(n \Delta t)^{\gamma-3}+(\gamma-3)(n \Delta t)^{\gamma-4} \Delta t+(n \Delta t)^{\gamma-5} O\left((\Delta t)^{2}\right)\left(\operatorname{resp} .((n+1) \Delta t)^{\gamma-3} \leq(n \Delta t)^{\gamma-3}\right)$.

From (4.2), we conclude that

$$
\begin{aligned}
& \sum_{j \in J_{N}} \int_{(j-1) \Delta x}^{(j+1) \Delta x}(N \Delta t)^{\gamma-3} F\left(x, N \Delta t, v_{j}^{N}\right) d x \\
& \leq \sum_{j \in J_{N_{1}}} \int_{(j-1) \Delta x}^{(j+1) \Delta x}\left(N_{1} \Delta t\right)^{\gamma-3} F\left(x, N_{1} \Delta t, v_{j}^{N_{1}}\right) d x+N \Delta t O(\Delta x) \\
& \leq \sum_{j \in J_{N_{1}}} \int_{(j-1) \Delta x}^{(j+1) \Delta x} F\left(x, N_{1} \Delta t, v_{j}^{N_{1}}\right) d x+N \Delta t O(\Delta x) \\
& \leq \sum_{j \in J_{0}} \int_{(j-1) \Delta x}^{(j+1) \Delta x} F\left(x, 0, v_{j}^{0}\right) d x+(3-\gamma) \frac{N_{1}\left(N_{1}-1\right)}{2}(\Delta t)^{2} \sum_{j \in J_{0}} \int_{(j-1) \Delta x}^{(j+1) \Delta x} \eta\left(x, 0, v_{j}^{0}\right) d x \\
& \quad+N \Delta t O(\Delta x) .
\end{aligned}
$$

Therefore, from (3.11) and (3.9), we obtain Lemma 4.1. 
Step 2. In this step, we derive (4.1). First we deduce from (3.3) the estimate of $\rho_{j}^{n}$ :

$$
\begin{aligned}
\sum_{j \in J_{n+1}} & \int_{(j-1) \Delta x}^{(j+1) \Delta x} x^{2} \rho_{j}^{n+1} d x \\
= & \sum_{j \in J_{n+1}} \int_{(j-1) \Delta x}^{(j+1) \Delta x} x^{2} \frac{\rho_{j+1}^{n}+\rho_{j-1}^{n}}{2} d x-\sum_{j \in J_{n+1}} \int_{(j-1) \Delta x}^{(j+1) \Delta x} x^{2} \frac{\Delta t}{2 \Delta x}\left(m_{j+1}^{n}-m_{j-1}^{n}\right) d x \\
= & \sum_{j \in J_{n+1}}\left\{\int_{(j+1) \Delta x}^{(j+3) \Delta x} x^{2} d x+\int_{(j-1) \Delta x}^{(j+1) \Delta x} x^{2} d x\right\} \frac{\rho_{j+1}^{n}}{2} \\
& +\sum_{j \in J_{n+1}}\left\{\int_{(j+1) \Delta x}^{(j+3) \Delta x} x^{2} d x-\int_{(j-1) \Delta x}^{(j+1) \Delta x} x^{2} d x\right\} \frac{\Delta t}{2 \Delta x} m_{j+1}^{n} \\
= & \sum_{j \in J_{n}} \frac{1}{2}\left\{\int_{j \Delta x}^{(j+2) \Delta x} x^{2} d x+\int_{(j-2) \Delta x}^{j \Delta x} x^{2} d x\right\} \rho_{j}^{n} \\
& +\sum_{j \in J_{n}}\left\{\int_{j \Delta x}^{(j+2) \Delta x} x^{2} d x-\int_{(j-2) \Delta x}^{j \Delta x} x^{2} d x\right\} \frac{\Delta t}{2 \Delta x} m_{j}^{n} \\
= & \sum_{j \in J_{n}} \frac{6 j^{2}+8}{3}(\Delta x)^{3} \rho_{j}^{n}+\sum_{j \in J_{n}} 4 j(\Delta x)^{2} \Delta t m_{j}^{n} \\
= & \sum_{j \in J_{n}} \int_{(j-1) \Delta x}^{(j+1) \Delta x} x^{2} \rho_{j}^{n} d x+\sum_{j \in J_{n}} \int_{(j-1) \Delta x}^{(j+1) \Delta x} 2 x \Delta t m_{j}^{n} d x+\sum_{j \in J_{n}} \int_{(j-1) \Delta x}^{(j+1) \Delta x}(\Delta x)^{2} \rho_{j}^{n} d x .
\end{aligned}
$$

Similarly we deduce from (3.4) the estimate of $m_{j}^{n}$ :

$$
\begin{aligned}
& \sum_{j \in J_{n+1}} \int_{(j-1) \Delta x}^{(j+1) \Delta x} 2 x(n+1) \Delta t m_{j}^{n+1} d x \\
& =\sum_{j \in J_{n}} \int_{(j-1) \Delta x}^{(j+1) \Delta x} 2 x(n+1) \Delta t m_{j}^{n} d x+\sum_{j \in J_{n}} \int_{(j-1) \Delta x}^{(j+1) \Delta x} 2(n+1)(\Delta t)^{2}\left\{\frac{\left(m_{j}^{n}\right)^{2}}{\rho_{j}^{n}}+p\left(\rho_{j}^{n}\right)\right\} d x
\end{aligned}
$$

Combining the two equations above and (3.10), we have

$$
\begin{aligned}
\sum_{j \in J_{n+1}} & \int_{(j-1) \Delta x}^{(j+1) \Delta x} F\left(x,(n+1) \Delta t, v_{j}^{n+1}\right) d x \\
\leq & \sum_{j \in J_{n}} \int_{(j-1) \Delta x}^{(j+1) \Delta x} F\left(x, n \Delta t, v_{j}^{n}\right) d x+\sum_{j \in J_{n}} \int_{(j-1) \Delta x}^{(j+1) \Delta x} \Delta t H\left(x, n \Delta t, v_{j}^{n}\right) d x \\
& +\sum_{j \in J_{n}} \int_{(j-1) \Delta x}^{(j+1) \Delta x}(\Delta x)^{2} \rho_{j}^{n} d x-\sum_{j \in J_{n}} \int_{(j-1) \Delta x}^{(j+1) \Delta x}(\Delta t)^{2}\left\{\frac{\left(m_{j}^{n}\right)^{2}}{\rho_{j}^{n}}+p\left(\rho_{j}^{n}\right)\right\} d x \\
& +\sum_{j \in J_{n}} \int_{(j-1) \Delta x}^{(j+1) \Delta x}(\Delta t)^{2}(1 / \theta-1) p\left(\rho_{j}^{n}\right) d x .
\end{aligned}
$$

Therefore, from (3.8), (3.10) and (3.11), we conclude (4.1). 
4.2. Estimates of $v^{\Delta}(x, t)$ in $N \Delta t \leq t<(N+1) \Delta t$. In this subsection, we prove the following lemma:

Lemma 4.2. For $T$ with $N \Delta \leq T<(N+1) \Delta t$, we have

$$
\int_{-\infty}^{\infty} T^{\gamma-3} F\left(x, T, v^{\Delta}(x, T)\right) d x \leq \sum_{j \in J_{N}} \int_{(j-1) \Delta x}^{(j+1) \Delta x}(N \Delta t)^{\gamma-3} F\left(x, N \Delta t, v_{j}^{N}\right) d x .
$$

This lemma follows from the following lemma:

Lemma 4.3. For $j \in J_{N+1}$, we have

$$
\begin{aligned}
& \int_{(j-1) \Delta x}^{(j+1) \Delta x} T^{\gamma-3} F\left(x, T, v^{\Delta}(x, T)\right) d x \\
& \quad \leq \frac{\int_{j \Delta x}^{(j+1) \Delta x}(N \Delta t)^{\gamma-3} F\left(x, N \Delta t, v_{j+1}^{N}\right) d x+\int_{(j-1) \Delta x}^{j \Delta x}(N \Delta t)^{\gamma-3} F\left(x, N \Delta t, v_{j-1}^{N}\right) d x}{2} \\
& \quad-\left(\int_{N \Delta t}^{T} t^{\gamma-3} G\left((j+1) \Delta x, t, v_{j+1}^{N}\right) d t-\int_{N \Delta t}^{T} t^{\gamma-3} G\left((j-1) \Delta x, t, v_{j-1}^{N}\right) d t\right) .
\end{aligned}
$$

Proof. We consider the region where $(j-1) \Delta x \leq x \leq(j+1) \Delta x$ and $N \Delta t \leq t \leq T$. Recall that $v^{\Delta}(x, t)$ is a Riemann solution in this region. For simplicity, we consider the case where one shock arises. Let the propagation speed of the shock be $\lambda$. Then the ray $x-j \Delta x=\lambda(t-N \Delta t)$ divides the region into two parts. On the other hand, if $v^{\Delta}(x, t)$ is a smooth solution to (1.1), we have

$$
\begin{aligned}
\left\{t^{\gamma-3} F\left(x, t, v^{\Delta}(x, t)\right)\right\}_{t}+\left\{t^{\gamma-3} G\left(x, t, v^{\Delta}(x, t)\right)\right\}_{x} & =-(3-\gamma) t^{\gamma-4}(x-t u)^{2} \rho \\
& \leq 0,
\end{aligned}
$$

because $\eta\left(v^{\Delta}\right)_{t}+q\left(v^{\Delta}\right)_{x}=0$. Since $v^{\Delta}(x, t)$ is continuous and piecewise smooth in each of the divided parts, by applying the divergence theorem to (4.4), we have

the left-hand side of (4.3)

$\leq$ the right-hand side of (4.3)

$$
\begin{aligned}
& +\int_{N \Delta t}^{T}\left[t^{\gamma-3}\left\{G\left(x(t), t, v^{\Delta}(x(t)+0, t)\right)-G\left(x(t), t, v^{\Delta}(x(t)-0, t)\right)\right\}\right. \\
& \left.-\lambda t^{\gamma-3}\left\{F\left(x(t), t, v^{\Delta}(x(t)+0, t)\right)-F\left(x(t), t, v^{\Delta}(x(t)-0, t)\right)\right\}\right] d t,
\end{aligned}
$$

where $x(t):=j \Delta x+\lambda(t-N \Delta t)$.

On the other hand, from (2.1) and (2.2), it follows that $v^{\Delta}(x, t)$ satisfies the following:

$$
\begin{aligned}
& f\left(v^{\Delta}(x(t)+0, t)\right)-f\left(v^{\Delta}(x(t)-0, t)\right)=\lambda\left\{v^{\Delta}(x(t)+0, t)-v^{\Delta}(x(t)-0, t)\right\}, \\
& q\left(v^{\Delta}(x(t)+0, t)\right)-q\left(v^{\Delta}(x(t)-0, t)\right) \leq \lambda\left\{\eta\left(v^{\Delta}(x(t)+0, t)\right)-\eta\left(v^{\Delta}(x(t)-0, t)\right)\right\} .
\end{aligned}
$$

Therefore we can prove the lemma.

By virtue of the compactness framework results of the approximate solutions (see 2, Section 4]), there exists a subsequence $v^{\Delta_{k}}$ such that $(\Delta x)_{k} \rightarrow 0$ and $v^{\Delta_{k}}$ tends to a 
weak solution to (1.3) almost everywhere as $k \rightarrow \infty$. On the other hand, we observe

$$
F(x, t, v)=(x-t u)^{2} \rho+t^{2} \rho^{\gamma} /(\gamma \theta) \geq t^{2} \rho^{\gamma} /(\gamma \theta) .
$$

Then, applying Lemmas 4.1 and 4.2 to the subsequence above, we conclude Theorem 1.1 .

Acknowledgments. The author would like to thank Professors S. Takeno and C. M. Dafermos for providing valuable suggestions and corrections.

\section{REFERENCES}

[1] A. Bressan, Hyperbolic Systems of Conservation Laws, (Oxford University Press, 2000). MR1816648 (2002d:35002)

[2] G.-Q. Chen, The compensated compactness method and the system of isentropic gas dynamics, MSRI preprint 00527-91, Berkeley (1990).

[3] X. Ding, G.-Q. Chen and P. Luo, Convergence of the Lax-Friedrichs scheme for isentropic gas dynamics (I)-(II), Acta Mathematica Scientia 7 (1987), 467-480, 8 (1988), 61-94 (in Chinese), 5 (1985), 415-432, 433-472 (in English). MR0922139 (89f:76006)

[4] B. Perthame, Kinetic formulation of conservation laws. Oxford Lecture Series in Mathematics and its Applications, 21., (Oxford University Press, Oxford, 2002). MR2064166

[5] J. Smoller, Shock Waves and Reaction-Diffusion Equations, (Springer-Verlag, New York, 1983). MR0688146(84d:35002) 